\title{
3 Research Square

\section{The association between serum uric acid and controlled attenuation parameter in United States adults without hepatitis B and C: a cross-sectional study}

\section{Mingming He}

The First Affiliated Hospital of Jiamusi University, Jiamusi, China

Lihong Chen ( $\square$ 464976459@qq.com )

First Affiliated Hospital of Anhui Medical University

\section{Yu Wang}

The First Affiliated Hospital of Jiamusi University, Jiamusi, China

\section{Haijun Ma}

The First Affiliated Hospital of Jiamusi University, Jiamusi, China

\section{Research Article}

Keywords: Controlled attenuation parameter, Serum uric acid, Hepatic steatosis, Transient elastography

Posted Date: April 21st, 2021

DOl: https://doi.org/10.21203/rs.3.rs-439398/v1

License: (c) (1) This work is licensed under a Creative Commons Attribution 4.0 International License.

Read Full License 


\section{Abstract}

Background: Controlled attenuation parameter (CAP) is a kind of widespread popular parameter to evaluate various types of hepatic steatosis by liver ultrasound transient elastography. We investigated the relationship between serum uric acid (SUA) and CAP without hepatitis B and C virus-infected in the United States adults, data from National Health and Nutrition Examination Survey (NHANES).

Methods: The present study was cross-sectional research. 4319 American men and women participants $\geq 18$ years old, without $B$ and $C$ hepatitis, were included in our analysis. There are some measures to evaluate the association between SUA and CAP by multivariate logistic regression analyses, fit smoothing curves, generalized additive models, two-piecewise linear regression model and subgroup analyses.

Results: There was a positive association between the value of SUA and CAP by multivariate logistic regression analyses after adjusting for various confounders. Besides, the inflection point of non-linear curve relationship was identified as $4.3 \mathrm{mg} / \mathrm{dL}$, for SUA $<4.3 \mathrm{mg} / \mathrm{dL}$, the effect size is $10.6(P<0.01)$; Correspondingly, SUA $\geq 4.3 \mathrm{mg} / \mathrm{dL}$, the effect size is $4.3(P<0.01)$.Finally, SUA was positively associated with glycohemoglobin less than $6.5 \%$ individuals $(\beta=7.3, P<0.01)$ and Fasting glucose less than 7.0 $\mathrm{mmol} / \mathrm{L}$ individuals $(\beta=6.8, P<0.01)$ in the subgroup analysis.

Conclusions: Our research found the relationship between SUA and CAP is non-linear. Subgroup analysis indicated that the positive association between SUA and CAP were showed in non-diabetic patients but not in diabetic.

\section{Background}

Hepatic steatosis, due to the unbalance of lipid metabolism in liver cells, various lipid substances appear in the cytoplasm of liver cells such as triglycerides, cholesterol etc. [1, 2]. The causes of hepatic steatosis include alcoholic, non-alcoholic, infected hepatitis B and C virus, etc. Among them, two main mortal reasons are the non-alcoholic and alcoholic fatty liver [3, 4]. In daily clinical practice, the paramount measure for diagnosing various kinds of fatty liver was pathologic findings, but its price and risk limited clinical application.

In non-invasive diagnostic methods, the value of controlled attenuation parameter (CAP) is a kind of widespread popular parameter to evaluate various types of hepatic steatosis for its convenience and accuracy, which measures ultrasound attenuation related to the presence of fatty liver tissue by liver ultrasound transient elastography $[5,6]$. Serum uric acid (SUA) is an end product from adenine and guanine nucleotides metabolism by related oxidase enzyme [7]. Correspondingly, a large amount of clinical evidence shows that elevated SUA is a marker of heart failure [8], insulin resistance [9], gout and nephrolithiasis [10]. 
The traditional ultrasound diagnosis result of fatty liver has confirmed to be related to SUA [11].As for assessing hepatic steatosis by ultrasound attenuation \Min-Sun Kwak et al [12] indicated the increase of CAP is likely to be a sign of early laboratory examination in liver steatosis with various abnormal metabolism of substances. However, the research that the association between SUA and CAP in the United States population infected without hepatitis $B$ and $C$ virus has limited. In our study, we analysed the data to evaluate the relationship between SUA and CAP without $B, C$ hepatitis population, data from NHANES of the American National Center for Health Statistics.

\section{Methods}

\section{Study population}

We executed a cross-sectional study, data from NHANES that has collected data about health status, diet type and disease-related examination, by a cross-sectional investigation in the sample of United States population since 1970s [13]. Our study analysis based on data from the 2017-2018 year represents a cycle of the NHANES. Of the 9254 participants, we excluded 3398 participants with age $\otimes 18$ years $\ 737$ participants missing CAP(controlled attenuation parameter) information, 299 participants missing

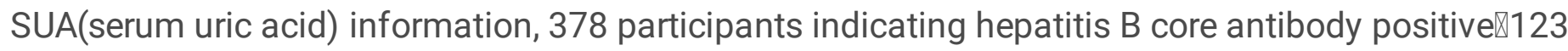
participants indicating hepatitis $\mathrm{C}$ antibody/cRNA positive or missing relative information about hepatitis C. Finally, in all of 4319 respondents $\geq 18$ years old, without B and C hepatitis, were selected in our investigation.

\section{Variables}

SUA was regarded as an exposure variable of the study. Roche Cobas 6000 Chemistry Analyzer (Roche Diagnostics Corporation, Indianapolis, IN 46250) used to measure SUA. Reagents were supplied by Roche Cat. No. 03183807190, UA2 reagent kit (400 tests). The outcome variable was the CAP (controlled attenuation parameter) used to assess hepatic steatosis. The value of CAP was acquired by FibroScan ${ }^{\circledR}$ using ultrasound and the vibration controlled transient elastography (VCTETM ). However, to ensure accuracy and security of the examination, individuals were excluded if they (1) were unable to cooperate with inspection, (2) were pregnant or unsure if no pregnant, (3) had an electronic medical device in the body, or (4) had lesions that cannot be checked.

\section{Covariates}

(1) Socio-demographic variables include age, gender, marital status, race and educational level. Except for age as continuous covariates, the others are all categorical variables. If participants were widowed, divorced, never married or separated were defined as single, while one participant refused to provide or missing information about marital status defined as other. (2) Smoking behaviour, alcohol intake and body mass index (BMI) are lifestyle variables defined as categorical variables. If an individual smoked more than 100 cigarettes in life, smoking behaviour defined as yes. Otherwise, it defined as no. The year before the survey, the amount of drink intake less than $17.01 \mathrm{~g}, 17.01 \mathrm{~g}-34.02 \mathrm{~g}$ and more than $34.02 \mathrm{~g}$ were 
defined as low, medium and high, respectively. (3) Clinical and laboratory examination include glycohemoglobin, fasting glucose, albumin(Alb), globulin, serum creatinine ( $\mathrm{SCr}$ ), alanine aminotransferase (ALT), gamma-glutamyl transaminase (GGT), lactate dehydrogenase (LDH), total bilirubin (TBi), high-density lipoprotein (HDL), cholesterol, liver stiffness measurement (LSM) and controlled attenuation parameter(CAP). Except for glycohemoglobin and fasting glucose as categorical variables, the others are continuous covariates. About the above variables and covariates, more details presented at https://www.cdc.gov/nchs/nhanes/.

\section{Statistical analysis}

Participants were categorized into quartiles according to SUA concentration. Descriptive characteristics of participants presented as percentages (categorical variables) and means \pm standard deviations (continuous variables). The weighted Chi-square test and the one-way ANOVA or Kruskal Wallis $\mathrm{H}$ test were performed to compare descriptive characteristics among quartiles of SUA. A univariate and multivariate weighted linear regression model was used to assess the relationship between SUA and CAP in different models. Crude model adjusted for none. Model I adjusted for gender, age, race. Model II adjusted for gender, age, race, education level, marital status, ALT, ALB, SCr, globulin, GGT, LDH, TBi, serum cholesterol, smoking behaviour, fasting glucose, HDL, LSM, alcohol intake, BMI, glycohemoglobin. Moreover, the non-linear relationship between SUA and CAP was found by smooth curve fittings and generalized additive models (GAM). The inflection point in the non-linear relationship was calculated using a two-piecewise linear regression model. Finally, subgroup analysis was performed to assess the association between SUA and CAP, and an interaction test was executed. All analysis use package R (http://www.Rproject.org) and EmpowerStats (http://www.empowerstats.com), with a $P$-value $<0.05$ defined as statistically significant.

\section{Results}

\section{Baseline characteristics of individuals}

The baseline characteristics of individuals according to quartiles of SUA are presented in Table 1. A total of 4319 participants, more than 18 years old, were included in our study, with those participants divided into four groups. There were notable differences in descriptive characteristics among the SUA quartiles, except race, education level, marital status, and globulin. Compared to the other groups (Q1-Q3), respondents located in the top group (Q4) of SUA were regarded to be male, black, and to have obesity $(B M I \geq 30)$, the behavior of smoking, alcohol intake. Furthermore, compared with the other three SUA groups (Q1-Q3), patients had a significantly higher glycohemoglobin, Alb, SCr, ALT, GGT, LDH, cholesterol, LSM, and CAP in the top groups (Q4).

\section{Univariate analysis for CAP}

The results of univariate analysis for controlled attenuation parameter (CAP) were shown in Table 2. We found that there were statistically significant in univariate analyses for CAP, with the exception of alcohol 
intake. Further, the results showed that BMI ( $\geq 30 \mathrm{~kg} / \mathrm{m} 2)$, glycohemoglobin ( $\geq 6.5 \%)$, fasting glucose ( $\geq 7.0 \mathrm{mmol} / \mathrm{L}$ ), Globulin, SCr, ALT, GGT, LDH, cholesterol, LSM, SUA were associated with higher CAP.

\section{Positive relationship between SUA and CAP}

The results of multivariate regression analysis after adjusting for various confounding factors were used to assess the relationship between SUA and CAP in different models, which are presented in Table 3 . In the Crude Model that was unadjusted for confounders, SUA was a positive association with CAP ( $\beta=$ 13.5, 95\% Cl: 12.2-14.7, $P<0.001)$. With simple and full adjustment for confounders, the association was also positive in model $\mathbb{\nabla}(\beta=11.8,95 \% \mathrm{Cl}: 10.4-13.2, P<0.001)$ and model $\mathbb{Z}(\beta=5.4,95 \% \mathrm{Cl}$ : $4.1-6.6, P<$ $0.001)$, respectively. After turning continuous variables SUA into categorical variables (quartiles), participants in the top of the serum uric acid group (Q4) had a $17.6 \mathrm{~dB} / \mathrm{m}(95 \% \mathrm{Cl}: 12.7,22.5, P<0.001)$ greater CAP than those in the bottom group. Besides, we performed a trend test, which is also statistically significant $(P<0.001)$.

\section{The analyses of non-linear relationship}

In the investigation, the non-linear relationship was examined between SUA and CAP by smooth curve fittings and generalized additive models, which are shown in Figure 1. We found the non-linear relationship between SUA and CAP after adjusting related confounders. What's more, the inflection point of curve was identified as $4.3 \mathrm{mg} / \mathrm{dL}$ (Table 4) with a two-piecewise linear regression model. For SUA $<4.3$ $\mathrm{mg} / \mathrm{dL}$, each additional $1 \mathrm{mg} / \mathrm{dL}$ SUA was associated with a $10.6 \mathrm{~dB} / \mathrm{m}$ increase in CAP. $(95 \% \mathrm{Cl}: 6.3-$ 14.8); However, SUA $\geq 4.3 \mathrm{mg} / \mathrm{dL}$, each additional $1 \mathrm{mg} / \mathrm{dL}$ SUA was only associated with a $4.3 \mathrm{~dB} / \mathrm{m}$ increase in CAP (95\% Cl: 2.9-5.8).

\section{Subgroup analysis}

Our subgroup analysis, stratified by age, gender, alcohol intake, smoking behavior, glycohemoglobin, and fasting glucose, as well as interaction test, showed in Table 5. Statistical significant interactions were detected between SUA and age, gender, glycohemoglobin, fasting glucose, respectively. Furthermore, it is surprising that SUA was positively associated with less than $6.5 \%$ group of glycohemoglobin $(\beta=7.3$, $95 \% \mathrm{Cl}$ : $6.0-8.6, P<0.001)$ and less than $7.0 \mathrm{mmol} / \mathrm{L}$ group of Fasting Glucose $(\beta=6.8,95 \% \mathrm{Cl}$ : 5.5- 8.0, $P<$ $0.001)$ in the subgroup analysis.

\section{Discussion}

Our study revealed the positive association between SUA and CAP in non-hepatitis B and non-hepatitis C population by multivariate regression analysis, even without excluding the alcohol intake population. After adjusted gender, age, race, education level, marital status, ALT, albumin, SCr, globulin, GGT, LDH, TBi, cholesterol, smoking behaviour, fasting glucose, HDL, LSM, alcohol intake, BMI and glycohemoglobin, CAP increased by $5.4 \mathrm{~dB} / \mathrm{m}$ stems from $1 \mathrm{mg} / \mathrm{dL}$ increase in SUA(95\%Cl: 4.1-6.6). Moreover, by a series of related non-linear relationship statistical model, we found the non-linear relationship between SUA and 
CAP, as well as calculated inflection point. According to the diagnostic criteria for diabetes, fasting glucose $\geq 7.0 \mathrm{mmol} / \mathrm{L}$ or glycohemoglobin $\geq 6.5 \%$ were diagnosed diabetes [14]. Consequently, with diabetes relative subgroup analysis, the relationship of SUA and CAP showed a positive association only in respondents with non-diabetes, but the difference between the two ways was not significant in patients with diabetes. However, in the subgroup analysis of alcohol intake, SUA and CAP showed a positive correlation in all subgroup $(p<0.001)$.

Searching for PubMed, we found a few articles that reported SUA and fatty liver showed a positive correlation. Roberto Catanzaro et al. [15] found a positive association between non-alcoholic fatty liver disease (NAFLD) and high concentration of SUA in the Mediterranean individual, and Xiaoya Zheng et al. [16] also reported the same relationship in non-obesity Chinese adults. However, they also used traditional abdominal ultrasound to evaluate NAFLD, which was lower accuracy than CAP $[17,18]$. Similarly, CAP and alcoholic hepatic steatosis were positively associated. Jan Petrasek et al.[19] proved that uric acid and ATP were regarded as taking part in an inflammatory reaction between hepatocytes and inflammatory cells and play a pivotal role in liver inflammation in vitro experiments with alcohol participation. Maja Thiele et al. [20] pointed that for alcoholic fatty liver, or other causes for a fatty liver had an excellent diagnostic precision with CAP, even severe steatosis can also be applied. Whereas, the notable disadvantage was that the research sample size was too small, and NAFLD was also excluded from his study.

In our research, we did not exclude either NAFLD or alcoholic fatty liver disease (AFLD) in the study individuals but executed a subgroup analysis to evaluate the effect of alcohol on the relationship between SUA concentration and the value of CAP. We found that SUA and CAP showed a positive association in each alcohol intake subgroup, and the effect size was statistically significant. Our research is similar to the Victor de Lédinghen et al. [21] result that the value of CAP was a significantly positive relationship with alcohol intake, which was attributed to the fatty liver diagnoses caused by drinking.

Ming-Hsiung Shih et al. [22] analysed 5370 participants from the NHANHES III to conclude that respondents with a higher concentration of SUA had a higher incidence of NAFLD \assessed by traditional ultrasound evaluation $\nabla$, and it was not affected by gender factors. However, in the 4002 population based Chinese cohort, Xianghai Zhou et al. [23] found a higher concentration SUA for only women independently correlated with higher odds of NAFLD(assessed by unenhanced abdominal CT), which is consistent with our research. Performing gender subgroup analysis and interaction test, we found that its interaction was statistically significant $(p<0.001)$, and SUA was positively associated with CAP in female, with an effect size of $9.3(95 \% \mathrm{Cl}: 7.4-11.2)$.

Our research has many advantages. Firstly, we use generalized additive models to evaluate SUA and CAP based on smooth curve fittings, considering that GAM can better predict their nonlinear relationship. Secondly, to make our research results stable and reliable, we used some sensitivity analysis, such as trend test and interaction test. Thirdly, compared with traditional ultrasound, we chose CAP as the 
outcome variable in the database, and it can not only qualitatively but also quantitatively diagnose hepatic steatosis, which makes the result more accurate[24, 25].

Our research also has some shortcomings. On the one hand, as a kind of observational study, the crosssectional research only reveals a positive association rather than discovers the causal link between SUA and CAP. On the other hand, this study only based on the study of the American population, and the application in other countries will be limited due to the factor that NHANES is a survey aimed at collect data on the disease and nutrition of the American [26].

\section{Conclusions}

Our research found the relationship between SUA and CAP is non-linear. Significantly, the positive association between SUA and CAP were showed in non-diabetic patients but not in diabetic.

\section{Abbreviations}

CAP: Controlled attenuation parameter; SUA: Serum uric acid; BMI: Body mass index; Alb: Albumin; SCr: Serum creatinine; ALT: Alanine aminotransferase; GGT: Gamma-glutamyl transaminase; LDH: Lactate dehydrogenase; TBi: Total bilirubin; HDL: High-density lipoprotein; LSM: Liver stiffness measurement; NAFLD: Non-alcoholic fatty liver disease; AFLD: Alcoholic fatty liver disease

\section{Declarations}

\section{Ethics approval and consent to participate}

All NHANES data acquisition is approved by the ethics review board of the National Center for Health Statistics.

\section{Consent for publication}

Not applicable.

\section{Availability of data and materials}

The datasets analysed during the current study are available in the NHANES repository, https://wwwn.cdc.gov/nchs/nhanes/Default.aspx.

\section{Competing interests}

The authors declare that they have no competing interests.

\section{Funding}

The writing of the manuscript was funded by scientific research project of Health and Family Planning Commission of Heilongjiang Province, No. 2019-3793. There are not roles in the design of the study and 
collection, analysis, interpretation of data of the manuscript.

\section{Authors' contributions}

$\mathrm{M} \mathrm{H}, \mathrm{L} \mathrm{C}$ contributed to the design, data collection, analysis, writing of this study. $\mathrm{Y} \mathrm{W}, \mathrm{H}$ M made revisions for related content. All authors read and approved the final manuscript.

\section{Acknowledgments}

Not applicable.

\section{Author details}

${ }^{1}$ General Surgery Department, The First Affiliated Hospital of Jiamusi University, Jiamusi, China.

${ }^{2}$ Department of Electrocardiography, The First Affiliated Hospital of Anhui Medical University, Hefei, China. ${ }^{3}$ Colorectal Surgery Department, The First Affiliated Hospital of Jiamusi University, Jiamusi, China.

\section{References}

1. Min H-K, Kapoor A, Fuchs M, Mirshahi F, Zhou H, Maher J, Kellum J, Warnick R, Contos Melissa J, Sanyal Arun J. Increased Hepatic Synthesis and Dysregulation of Cholesterol Metabolism Is Associated with the Severity of Nonalcoholic Fatty Liver Disease. Cell Metabolism. 2012; 15:665-74.

2. Cui A, Hu Z, Han Y, Yang Y, Li Y. Optimized Analysis of In Vivo and In Vitro Hepatic Steatosis. Journal of visualized experiments : JoVE. 2017.

3. You Y, Li WZ, Zhang S, Hu B, Li YX, Li HD, Tang HH, Li QW, Guan YY, Liu LX et al. SNX10 mediates alcohol-induced liver injury and steatosis by regulating the activation of chaperone-mediated autophagy. Journal of hepatology. 2018; 69:129-41.

4. Fuster D, Longo DL, Samet JH. Alcohol Use in Patients with Chronic Liver Disease. New England Journal of Medicine. 2018; 379:1251-61.

5. Kwok R, Choi KC, Wong GL, Zhang Y, Chan HL, Luk AO, Shu SS, Chan AW, Yeung MW, Chan JC et al. Screening diabetic patients for non-alcoholic fatty liver disease with controlled attenuation parameter and liver stiffness measurements: a prospective cohort study. Gut. 2016; 65:1359-68.

6. Moreno C, Mueller S, Szabo G. Non-invasive diagnosis and biomarkers in alcohol-related liver disease. Journal of hepatology. 2019; 70:273-83.

7. Selvaraj S, Claggett BL, Pfeffer MA, Desai AS, Mc Causland FR, McGrath MM, Anand IS, Veldhuisen DJ, Kober L, Janssens $S$ et al. Serum uric acid, influence of sacubitril-valsartan, and cardiovascular outcomes in heart failure with preserved ejection fraction: PARAGON-HF. European Journal of Heart Failure. 2020; 22:2093-101.

8. Huang G, Qin J, Deng X, Luo G, Yu D, Zhang M, Zhou S, Wang L. Prognostic value of serum uric acid in patients with acute heart failure: A meta-analysis. Medicine. 2019; 98:e14525. 
9. Mazidi M, Katsiki N, Mikhailidis DP, Banach M. The link between insulin resistance parameters and serum uric acid is mediated by adiposity. Atherosclerosis. 2018; 270:180-86.

10. Li X, Meng X, Timofeeva M, Tzoulaki I, Tsilidis KK, loannidis JP, Campbell H, Theodoratou E. Serum uric acid levels and multiple health outcomes: umbrella review of evidence from observational studies, randomised controlled trials, and Mendelian randomisation studies. Bmj. 2017; 357:j2376.

11. Lombardi R, Pisano G, Fargion S. Role of Serum Uric Acid and Ferritin in the Development and Progression of NAFLD. International Journal of Molecular Sciences. 2016; 17:548.

12. Kwak M-S, Chung GE, Yang JI, Yim JY, Chung SJ, Jung SY, Kim JS. Clinical implications of controlled attenuation parameter in a health check-up cohort. Liver International. 2018; 38:915-23.

13. Ahluwalia N, Dwyer J, Terry A, Moshfegh A, Johnson C. Update on NHANES Dietary Data: Focus on Collection, Release, Analytical Considerations, and Uses to Inform Public Policy. Advances in nutrition. 2016; 7:121-34.

14. 2. Classification and Diagnosis of Diabetes: Standards of Medical Care in Diabetes-2019. Diabetes Care. 2018; 42:S13-S28.

15. Catanzaro R, Sciuto M, He F, Singh B, Marotta F. Non-alcoholic fatty liver disease: correlation with hyperuricemia in a European Mediterranean population. Acta clinica Belgica. 2020:1-6.

16. Zheng X, Gong L, Luo R, Chen H, Peng B, Ren W, Wang Y. Serum uric acid and non-alcoholic fatty liver disease in non-obesity Chinese adults. Lipids in health and disease. 2017; 16:202.

17. Castera L, Friedrich-Rust M, Loomba R. Noninvasive Assessment of Liver Disease in Patients With Nonalcoholic Fatty Liver Disease. Gastroenterology. 2019; 156:1264-81 e4.

18. Xu L, Lu W, Li P, Shen F, Mi YQ, Fan JG. A comparison of hepatic steatosis index, controlled attenuation parameter and ultrasound as noninvasive diagnostic tools for steatosis in chronic hepatitis B. Digestive and liver disease : official journal of the Italian Society of Gastroenterology and the Italian Association for the Study of the Liver. 2017; 49:910-17.

19. Petrasek J, Iracheta-Vellve A, Saha B, Satishchandran A, Kodys K, Fitzgerald KA, Kurt-Jones EA, Szabo G. Metabolic danger signals, uric acid and ATP, mediate inflammatory cross-talk between hepatocytes and immune cells in alcoholic liver disease. Journal of leukocyte biology. 2015; 98:24956.

20. Thiele M, Rausch V, Fluhr G, Kjaergaard M, Piecha F, Mueller J, Straub BK, Lupsor-Platon M, DeLedinghen V, Seitz HK et al. Controlled attenuation parameter and alcoholic hepatic steatosis: Diagnostic accuracy and role of alcohol detoxification. Journal of hepatology. 2018; 68:1025-32.

21. de Ledinghen V, Vergniol J, Capdepont M, Chermak F, Hiriart JB, Cassinotto C, Merrouche W, Foucher J, Brigitte le B. Controlled attenuation parameter (CAP) for the diagnosis of steatosis: a prospective study of 5323 examinations. Journal of hepatology. 2014; 60:1026-31.

22. Shih M-H, Lazo M, Liu S-H, Bonekamp S, Hernaez R, Clark JM. Association between serum uric acid and nonalcoholic fatty liver disease in the US population. Journal of the Formosan Medical Association. 2015; 114:314-20. 
23. Zhou X, Li Y, Zhang X, Guan YY, Puentes Y, Zhang F, Speliotes EK, Ji L. Independent markers of nonalcoholic fatty liver disease in a gentrifying population-based Chinese cohort. Diabetes/Metabolism Research and Reviews. 2019; 35.

24. Rout G, Kedia S, Nayak B, Yadav R, Das P, Acharya SK, Gunjan D, Singh V, Mahanta M, Gupta S et al. Controlled Attenuation Parameter for Assessment of Hepatic Steatosis in Indian Patients. Journal of clinical and experimental hepatology. 2019; 9:13-21.

25. Mikolasevic I, Orlic L, Franjic N, Hauser G, Stimac D, Milic S. Transient elastography (FibroScan((R))) with controlled attenuation parameter in the assessment of liver steatosis and fibrosis in patients with nonalcoholic fatty liver disease - Where do we stand? World journal of gastroenterology. 2016; 22:7236-51.

26. Fain JA. Nhanes. The Diabetes educator. 2017; 43:151.

\section{Tables}

Table 1 Baseline characteristics of participants $(\mathrm{N}=4319)$. 


\begin{tabular}{|c|c|c|c|c|c|}
\hline \multirow[t]{2}{*}{ Characteristic } & \multicolumn{4}{|c|}{ Serum uric acid Quartiles (mg/dL) } & \multirow[t]{2}{*}{$P$ value } \\
\hline & Q1 & Q2 & Q3 & Q4 & \\
\hline No. of participants & 1035 & 1055 & 1067 & 1162 & \\
\hline Age (years, mean \pm sd) & $45.24 \pm 16.63$ & $44.28 \pm 17.27$ & $48.95 \pm 17.42$ & $48.16 \pm 18.18$ & $<0.0001$ \\
\hline Gender (\%) & & & & & $<0.0001$ \\
\hline Male & 14.80 & 39.08 & 60.74 & 77.73 & \\
\hline Female & 85.20 & 60.92 & 39.26 & 22.27 & \\
\hline Race (\%) & & & & & 0.0759 \\
\hline Mexican American & 10.45 & 9.20 & 9.25 & 9.55 & \\
\hline Other Hispanic & 8.01 & 7.29 & 6.64 & 5.98 & \\
\hline Non-Hispanic White & 63.19 & 63.89 & 66.53 & 61.37 & \\
\hline Non-Hispanic Black & 10.41 & 9.81 & 8.90 & 11.35 & \\
\hline Other Race & 7.95 & 9.81 & 8.69 & 11.76 & \\
\hline Marital status (\%) & & & & & 0.1955 \\
\hline Married or living with partner & 62.89 & 57.44 & 60.44 & 60.99 & \\
\hline Single & 33.75 & 38.47 & 36.69 & 35.66 & \\
\hline Other & 3.36 & 4.08 & 2.87 & 3.36 & \\
\hline Education level (\%) & & & & & 0.0893 \\
\hline Below high school education & 9.96 & 9.37 & 9.15 & 10.64 & \\
\hline High school graduate & 22.89 & 26.55 & 29.25 & 24.99 & \\
\hline College graduate & 63.78 & 59.99 & 58.64 & 60.98 & \\
\hline Not recorded & 3.36 & 4.09 & 2.96 & 3.39 & \\
\hline BMI (\%) & & & & & $<0.0001$ \\
\hline$<30\left(\mathrm{~kg} / \mathrm{m}^{2}\right)$ & 72.74 & 61.89 & 52.05 & 43.61 & \\
\hline$\geq 30$ & 27.26 & 38.11 & 47.95 & 56.39 & \\
\hline Smoking behavior (\%) & & & & & $<0.0001$ \\
\hline Yes & 33.84 & 40.59 & 44.12 & 43.24 & \\
\hline No & 66.16 & 59.41 & 55.88 & 56.76 & \\
\hline Alcohol intake (\%) & & & & & $<0.0001$ \\
\hline Low & 28.35 & 29.17 & 24.52 & 24.73 & \\
\hline Medium & 26.76 & 22.80 & 19.80 & 20.44 & \\
\hline High & 44.89 & 48.03 & 55.68 & 54.83 & \\
\hline Glycohemoglobin (\%) & & & & & 0.0080 \\
\hline$<6.5(\%)$ & 92.49 & 90.66 & 90.90 & 88.19 & \\
\hline
\end{tabular}




$$
\geq 6.5
$$

Fasting glucose (\%)

$$
\begin{aligned}
& <7.0(\mathrm{mmol} / \mathrm{L}) \\
& \geq 7.0
\end{aligned}
$$

Alb $(g / L$, mean $\pm \mathrm{sd})$

Globulin $(\mathrm{g} / \mathrm{L}$, mean $\pm \mathrm{sd})$

$\mathrm{SCr}(\mathrm{umol} / \mathrm{L}$, mean $\pm \mathrm{sd})$

ALT (IU/L, mean \pm sd)

GGT (IU/L, mean \pm sd)

$\mathrm{LDH}(\mathrm{IU} / \mathrm{L}$, mean $\pm \mathrm{sd})$

TBi (umol/L, mean \pm sd)

HDL $(\mathrm{mmol} / \mathrm{L}$, mean $\pm \mathrm{sd})$

Cholesterol $(\mathrm{mmol} / \mathrm{L}$, mean $\pm \mathrm{sd})$

LSM (kpa, mean \pm sd)

$\mathrm{CAP}(\mathrm{dB} / \mathrm{m}$, mean $\pm \mathrm{sd})$

7.51

9.34

9.10

11.81

0.0010

95.61

96.33

92.84

93.95

4.39

3.67

7.16

6.05

$\begin{array}{ccccc}40.57 \pm 3.03 & 40.86 \pm 3.29 & 41.27 \pm 3.08 & 41.36 \pm 3.27 & <0.0001 \\ 29.93 \pm 3.95 & 29.93 \pm 3.95 & 29.83 \pm 4.05 & 29.95 \pm 4.03 & 0.8887 \\ 66.48 \pm 27.83 & 72.88 \pm 19.69 & 80.15 \pm 19.75 & 91.12 \pm 43.23 & <0.0001 \\ 18.09 \pm 12.55 & 20.61 \pm 17.00 & 23.50 \pm 13.67 & 28.70 \pm 21.01 & <0.0001 \\ 23.77 \pm 35.02 & 24.86 \pm 31.06 & 28.73 \pm 27.16 & 37.70 \pm 53.24 & <0.0001 \\ 152.94 \pm 29.16 & 155.82 \pm 32.20 & 157.76 \pm 30.12 & 159.54 \pm 32.99 & <0.0001 \\ 6.90 \pm 3.97 & 7.53 \pm 4.36 & 8.94 \pm 5.60 & 8.72 \pm 4.69 & <0.0001 \\ 1.52 \pm 0.38 & 1.44 \pm 0.40 & 1.32 \pm 0.36 & 1.26 \pm 0.37 & <0.0001 \\ 4.80 \pm 0.97 & 4.88 \pm 1.06 & 4.89 \pm 1.02 & 4.97 \pm 1.08 & 0.0022 \\ 4.98 \pm 3.21 & 5.55 \pm 4.99 & 5.91 \pm 5.40 & 6.68 \pm 5.94 & <0.0001 \\ 239.24 \pm 57.55 & 253.91 \pm 60.73 & 274.44 \pm 62.01 & 286.39 \pm 63.56 & <0.0001\end{array}$

Abbreviations: BMI, body mass index; Alb, albumin; SCr, serum creatinine; ALT, alanine aminotransferase; GGT, gamma-glutamyl transaminase; LDH, lactate dehydrogenase; TBi, total bilirubin; HDL, high-density lipoprotein; LSM, liver stiffness measurement; CAP, controlled attenuation parameter.

Table 2: Univariate analysis for controlled attenuation parameter. 


\begin{tabular}{lclr}
\hline & Statistics, $\mathrm{n}(\%)$ & $\beta(95 \% \mathrm{CI})$ & $P$-value \\
\hline $\begin{array}{l}\text { Agelyear[ } \\
\text { Gender }\end{array}$ & $48.8 \pm 18.6$ & $0.8(0.7,0.9)$ & $<0.001$ \\
Male & $2088(48.3 \%)$ & Reference & \\
Female & $2231(51.7 \%)$ & $-21.0(-24.7,-17.2)$ & $<0.001$ \\
Race & & & \\
Mexican American & $656(15.2 \%)$ & Reference & \\
Other Hispanic & $421(9.7 \%)$ & $-22.1(-31.5,-12.7)$ & $<0.001$ \\
Non-Hispanic White & $1571(36.4 \%)$ & $-14.9(-21.4,-8.3)$ & $<0.001$ \\
Non-Hispanic Black & $920(21.3 \%)$ & $-29.1(-37.6,-20.6)$ & $<0.001$ \\
Other Race & $751(17.4 \%)$ & $-16.0(-24.6,-7.4)$ & $<0.001$
\end{tabular}

Marital status

Married or Living with partner

Single

2417 (56.0\%) Reference

Other

$1677(38.8 \%) \quad-17.6(-21.6,-13.7)<0.001$

$225(5.2 \%) \quad-48.1(-58.5,-37.7)<0.001$

BMI $\left(\mathrm{kg} / \mathrm{m}^{2}\right)$

$$
\begin{aligned}
& <30 \\
& \geq 30
\end{aligned}
$$

2510 (58.1\%) Reference

$1809(41.9 \%) \quad 62.4(59.0,65.8) \quad<0.001$

Smoking behavior

Yes

No

1723 (39.9\%) Reference

$2596(60.1 \%) \quad-13.9(-17.8,-10.1)<0.001$

Alcohol intake

Low

1053 (24.4\%) Reference

Medium

$843(19.5 \%) \quad-3.6(-9.1,1.8)$

0.191

High

$2423(56.1 \%)$

$3.5(-1.0,8.1)$

0.126

Education level

Below high school education

High school graduate

$\begin{array}{clc}769(17.8 \%) & \text { Reference } & \\ 979(22.7 \%) & 3.1(-4.0,10.2) & 0.392 \\ 2343(54.2 \%) & -4.8(-11.3,1.7) & 0.149 \\ 228(5.3 \%) & -44.2(-56.0,-32.4) & <0.001\end{array}$


Glycohemoglobin (\%)

$<6.5$

$\geq 6.5$

Fasting glucose(mmol/L)

$$
\begin{aligned}
& <7.0 \\
& \geq 7.0
\end{aligned}
$$

Albumin (g/L)

Globulin (g/L)

Scr (umol/L)

$\mathrm{ALT}(\mathrm{IU} / \mathrm{L})$

GGT (IU/L)

$\mathrm{LDH}(\mathrm{IU} / \mathrm{L})$

TBi (umol/L)

Cholesterol (mmol/L)

HDL (mmol/L)

LSM(Kpa)

SUA (mg/dL)
3732 (86.4\%) Reference

$587(13.6 \%) \quad 59.7(53.5,66.0) \quad<0.001$

$\begin{array}{cll}4000(92.6 \%) & \text { Reference } & \\ 319(7.4 \%) & 56.0(47.7,64.3) & <0.001 \\ 40.7 \pm 3.3 & -2.5(-3.1,-1.9) & <0.001 \\ 30.8 \pm 4.3 & 1.4(1.0,1.9) & <0.001 \\ 79.3 \pm 41.4 & 0.1(0.0,0.2) & 0.003 \\ 22.2 \pm 17.2 & 1.1(1.0,1.2) & <0.001 \\ 30.5 \pm 42.8 & 0.3(0.3,0.4) & <0.001 \\ 157.9 \pm 33.6 & 0.2(0.1,0.2) & <0.001 \\ 7.9 \pm 4.7 & -0.5(-0.9,-0.1) & 0.008 \\ 4.8 \pm 1.1 & 5.3(3.5,7.1) & <0.001 \\ 1.4 \pm 0.4 & -52.9(-57.5,-48.3) & <0.001 \\ 6.0 \pm 5.3 & 3.4(3.0,3.7) & <0.001 \\ 5.4 \pm 1.5 & 13.5(12.2,14.7) & <0.001\end{array}$

Abbreviations: BMI, body mass index; Alb, albumin; SCr, serum creatinine; ALT, alanine aminotransferase; GGT, gamma-glutamyl transaminase; LDH, lactate dehydrogenase; TBi, total bilirubin; HDL, high-density lipoprotein; LSM, liver stiffness measurement; SUA, serum uric acid.

Table 3 Relationship between SUA and controlled attenuation parameter in different

\begin{tabular}{|c|c|c|c|c|c|c|}
\hline \multirow[t]{2}{*}{ Variable } & \multicolumn{2}{|c|}{ Crude Model } & \multicolumn{2}{|l|}{ Model \ } & \multicolumn{2}{|l|}{ Model $\square$} \\
\hline & $\beta(95 \% \mathrm{CI})$ & $P$-value & $\beta(95 \% \mathrm{CI})$ & $P$-value & $\beta(95 \% \mathrm{CI})$ & $P$-value \\
\hline SUA & $13.5(12.2,14.7)$ & $<0.001$ & $11.8(10.4,13.2)$ & $<0.001$ & $5.4(4.1,6.6)$ & $<0.001$ \\
\hline \multicolumn{7}{|c|}{ SUA(quartile) } \\
\hline Q1 & Reference & & Reference & & Reference & \\
\hline Q2 & $14.7(9.5,19.8)$ & $<0.001$ & $13.8(8.7,18.9)$ & $<0.001$ & $6.5(2.3,10.7)$ & 0.002 \\
\hline Q3 & $35.2(30.1,40.4)$ & $<0.001$ & $29.0(23.6,34.3)$ & $<0.001$ & $14.7(10.2,19.3)$ & $<0.001$ \\
\hline Q4 & $47.2(42.0,52.3)$ & $<0.001$ & $40.4(34.8,46.1)$ & $<0.001$ & $17.6(12.7,22.5)$ & $<0.001$ \\
\hline$P$ for trend & $<0.001$ & & $<0.001$ & & $<0.001$ & \\
\hline
\end{tabular}
models. 
Abbreviations: CI, confidence interval; SUA, serum uric acid.

Crude model adjust for none. Model I adjust for gender, age, race. Model II adjust for gender, age, race, education level, marital status, alanine aminotransferase, albumin, serum creatinine, globulin, gamma-glutamyl transaminase, lactate dehydrogenase, total bilirubin, serum cholesterol, smoking behavior, fasting glucose, HDL-cholesterol, liver stiffness measurement, alcohol intake, body mass index, glycohemoglobin.

Table 4 Threshold effect analysis of serum uric acid and controlled attenuation parameter using a Piece-wise Linear Regression.

\begin{tabular}{ccccc}
\hline Inflection point $(\mathrm{mg} / \mathrm{dL})$ & Effect size $(\beta)$ & $95 \% \mathrm{CI}$ & $P$ value & log likelihood ratio \\
\hline$<4.3$ & 10.6 & 6.3 to 14.8 & $<0.001$ & 0.012 \\
$\geq 4.3$ & 4.3 & 2.9 to 5.8 & $<0.001$ & \\
\hline
\end{tabular}

Abbreviations: CI, confidence interval

Gender, age, race, marital status, alanine aminotransferase, albumin, serum creatinine, globulin, gamma-glutamyl transaminase, lactate dehydrogenase, total bilirubin, serum cholesterol, smoking behavior, liver stiffness measurement, HDL-cholesterol, alcohol intake, education level, body mass index, glycohemoglobin, fasting glucose were adjusted.

Table 5 Effect size of serum uric acid and controlled attenuation parameter in prespecified and exploratory subgroups in each subgroup 


\begin{tabular}{lcccc}
\hline & $\mathrm{N}$ & $\beta(95 \% \mathrm{CI})$ & $P$-value & $P$ for interaction \\
\hline Age (year) & & & & 0.0467 \\
$\quad<60$ & 2834 & $6.6(5.1,8.1)$ & $<0.001$ & \\
$\geq 60$ & 1485 & $3.9(1.9,5.9)$ & $<0.001$ & \\
Gender & & & & 0.0002 \\
$\quad$ Male & 2088 & $3.5(1.7,5.3)$ & $<0.001$ & \\
Female & 2231 & $9.3(7.4,11.2)$ & $<0.001$ & \\
Smoking behavior & & & & 0.0445 \\
$\quad$ Yes & 1723 & $4.7(2.7,6.7)$ & $<0.001$ & \\
$\quad$ No & 2596 & $7.5(5.9,9.0)$ & $<0.001$ & \\
Alcohol intake & & & & \\
Low & 1053 & $6.0(3.5,8.5)$ & $<0.001$ & \\
Medium & 843 & $7.8(5.0,10.7)$ & $<0.001$ & \\
High & 2423 & $5.8(4.2,7.4)$ & $<0.001$ & \\
Glycohemoglobin (\%) & & & & \\
$\quad<6.5$ & 3732 & $7.3(6.0,8.6)$ & $<0.001$ & \\
$\geq 6.5$ & 587 & $2.0(-0.8,4.8)$ & 0.162 & \\
Fasting glucose (mmol/L) & & & & \\
$\quad<7.0$ & 4000 & $6.8(5.5,8.0)$ & $<0.001$ & \\
$\geq 7.0$ & 319 & $0.6(-3.4,4.5)$ & 0.784 & \\
\hline
\end{tabular}

Abbreviations: CI, confidence interval.

Above model adjusted for race, education level, marital status, alanine aminotransferase, albumin, serum creatinine, globulin, gamma-glutamyl transaminase, lactate dehydrogenase, total bilirubin, serum cholesterol, HDL-cholesterol, liver stiffness measurement and body mass index except the subgroup variable.

\section{Figures}




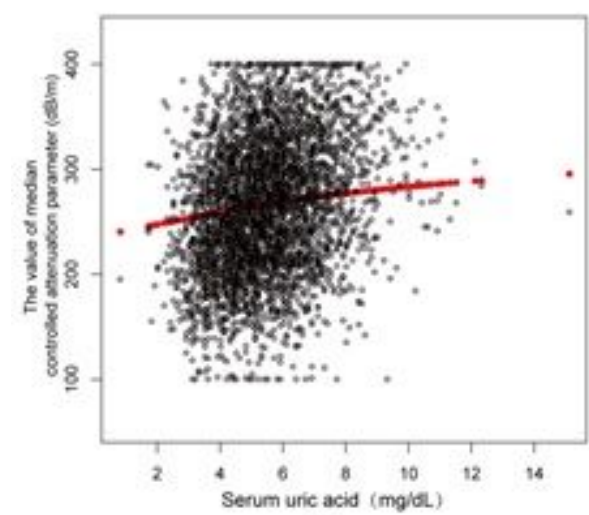

(a)

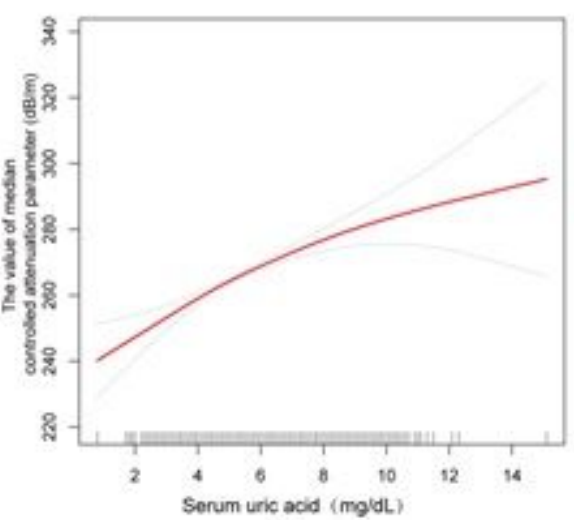

(b)

\section{Figure 1}

The positive association between serum uric acid (SUA) and controlled attenuation parameter (CAP). A threshold, nonlinear association between SUA and CAP was found (P凶0.001) in a generalized additive model (GAM). The solid red line shows the smooth curve fit between variables. The blue dotted line indicates the $95 \%$ of confidence interval. Gender, age, race, marital status, alanine aminotransferase, albumin, serum creatinine, globulin, gamma-glutamyl transaminase, lactate dehydrogenase, total bilirubin, serum cholesterol, smoking behavior, liver stiffness measurement, HDL-cholesterol, alcohol intake, education level, body mass index, glycohemoglobin, fasting glucose were adjusted. 